\title{
A systematic review of interventions aimed at improving the cardiovascular health of people diagnosed with personality disorders
}

\author{
Katherine Hall ${ }^{1}$ [D $\cdot$ Kirsten Barnicot ${ }^{2} \cdot$ Mike Crawford $^{2} \cdot$ Paul Moran $^{1}$ \\ Received: 3 December 2018 / Accepted: 26 March 2019 / Published online: 30 March 2019 \\ (c) The Author(s) 2019
}

\begin{abstract}
Purpose People with personality disorders have significantly reduced life expectancy and increased rates of cardiovascular disease compared to members of the general population. Given that more people die annually of cardiovascular disease across the globe than from any other cause, it is important to identify the evidence for interventions aimed at improving cardiovascular health among people with personality disorders.

Methods Systematic literature review. PsycINFO, MEDLINE and EMBASE were searched using NICE Healthcare Databases, as well as CENTRAL and trial registries. We sought to identify randomised controlled trials of interventions pertaining to adults with a primary diagnosis of personality disorder, where the primary outcome measure was cardiovascular health before and after the intervention.

Results A total of 1740 records were identified and screened by two independent reviewers. No papers meeting the inclusion criteria were identified.

Conclusions This systematic review did not identify any randomised controlled trials testing interventions aimed at improving the cardiovascular health of people with personality disorders. Research in this area could have important public health implications, spanning the fields of psychiatry and general medicine.
\end{abstract}

Keywords Personality disorders $\cdot$ Cardiovascular health $\cdot$ Systematic review

\section{Background}

Personality disorders (PDs) are complex mental health problems, with an overall estimated prevalence in western European countries of approximately $6 \%$ [1], affecting as many as $40 \%$ of those in contact with secondary mental health services [2]. The Diagnostic and Statistical Manual of Mental Disorders (DSM) classification of personality disorders into three clusters is widely used; Cluster A includes the paranoid, schizoid and schizotypal categories, Cluster B includes the antisocial, borderline, histrionic and narcissistic categories, and Cluster $\mathrm{C}$ includes the avoidant, dependent and obsessive-compulsive categories [3]. Although this

Katherine Hall

kh9743@my.bristol.ac.uk

1 Population Health Sciences, University of Bristol, Bristol, UK

2 Imperial College London, London, UK review aims to include people with any type of personality disorder, much of the relevant epidemiological research has been conducted in people with Cluster B PDs, and specifically borderline personality disorder (BPD).

People with PD have a lower average life expectancy than the general population, with figures ranging from 18 years fewer in a London-based retrospective cohort study [4] to as many as 27.5 years for people with severe PD in a Scottish cohort [5]. This loss of life years cannot simply be attributed to excess rates of suicide [6,7]. A recent review of the literature relating to physical health problems experienced by people with PD has summarised associations with pain conditions, obesity and chronic illnesses including CVDs [8]. The authors of this review found that, compared to controls, people diagnosed with Cluster B PDs were at increased risk of experiencing syncope, seizures and arthritis, as well as elevated levels of health concern in general [8]. It was also noted that people with PD experience faster annual rates of health decline (by 50\%) from adolescence to their mid-30 s compared to controls [8]. These health problems 
are associated with increased health service utilisation compared to the general population $[9,10]$. Other research has found differences in hospitalisation rates among people with borderline PD compared to controls which were especially apparent in relation to cardiac and gynaecological conditions [11]. ${ }^{1}$

According to the World Health Organization (WHO), more people die annually from cardiovascular diseases (CVDs) across the globe than from any other cause. CVDs include coronary heart disease, cerebrovascular disease and peripheral arterial disease [12]. In addition to the wellknown occurrence of psychiatric co-morbidities in people with PD [13], the disproportionately high burden of physical health problems, such as CVDs, is becoming increasingly recognised as contributory to the stark reduction in life expectancy in this group [4, 14-16]. Moreover, the finding that physical illnesses predict death by suicide illustrates the critical interplay between poor physical health and suicide among people with PD [13, 17, 18]. Evidence for an association between PD and CVDs comes from various population-based epidemiological studies, which, for BPD, demonstrate adjusted odds ratios for CVD ranging from 1.47 to 7.2 [19-21]. In a UK household survey $(n=8580)$, Moran et al. found that people with a PD were almost twice as likely to report a history of stroke, and around 1.4 times as likely to report a history of ischaemic heart disease compared to the general population, after adjustment for age, sex, socioeconomic status, hypertension, diabetes, smoking and alcohol use [21]. Similar figures come from the National Epidemiologic Survey on Alcohol and Related Conditions (NESARC) $(n=34,653)$, conducted in the United States, in which older adults with any PD were 1.26 times more likely to report a history of coronary heart disease than controls [22]. The longitudinal Baltimore study, in which 244 people with PD were followed up over a 23-year period, found that Cluster B disorders were consistently associated with increased CVD incidence, and that their presence predicted CVD mortality [20]. Furthermore, in a small sample of women with BPD $(n=47)$, common carotid artery ultrasound scanning demonstrated greater intima-media thickness (compared to healthy controls), an early marker for atherosclerosis and subsequent CVD risk [23].

When considering the psychopathology associated with $\mathrm{PD}$, the susceptibility to certain cardiovascular risk factors is perhaps unsurprising. People with BPD, with its association with early life adversity and tendency towards impulsivity, have been found to have an increased risk of metabolic syndrome and dysregulated glucose and lipid metabolism [24],

\footnotetext{
1 The authors do note that the increased presentation to cardiology departments may be confounded by the co-occurrence of anxiety disorders in patients with BPD.
}

as well as higher rates of obesity $[9,25]$. Interestingly and perhaps intuitively, rates of smoking, alcohol consumption and lack of regular exercise have been found to be higher in non-recovered patients with BPD compared to those who had recovered [9]. An Australian cross-sectional study of young community-dwelling adults $(n=1520)$ also found strong independent associations between Cluster B PDs and smoking [26].

The WHO, like many national-level organisations, emphasises that most CVDs can be prevented by addressing behavioural risk factors such as tobacco smoking, poor diet, obesity and lack of exercise [12]. These factors, as well as elevated blood glucose levels and hyperlipidaemia, are recognised as being causally linked to CVD [27]. However, it cannot be assumed that the usual CVD risk reduction strategies are generalisable to people with PD; in this group, compared to the general population, important differences regarding patterns of healthcare utilisation [28], treatment adherence [29], and co-morbidities [30] must all be considered. We chose to carry out this systematic review as targeted interventions in this area are likely to provide an important avenue to narrow the health and life expectancy gap faced by people with PDs.

\section{Objectives}

1. To assess whether any interventions designed to improve the cardiovascular health or cardiovascular risk factors in people diagnosed with PDs have been developed and evaluated using randomised controlled trials (RCTs).

2. To assess whether any such interventions are efficacious.

\section{Methods}

\section{Search strategy and selection criteria}

This systematic review was conducted in accordance with the Preferred Reporting Items for Systematic Reviews and Meta-Analyses (PRISMA) guidelines [31].

We searched for RCTs of interventions aimed at improving cardiovascular disease or improving cardiovascular risk factors in people with a primary diagnosis of PD.

\section{Inclusion criteria}

Trials were eligible for inclusion if they met the following criteria: [1] used random allocation to assign participants to the intervention and control groups; [2] all participants were adults (aged 18-65 years) with a primary diagnosis of any type of PD, irrespective of the diagnostic criteria used. 
We set out to include studies of any intervention aimed at improving cardiovascular disease or improving cardiovascular risk factors. This would include studies aimed at promoting smoking cessation, increasing levels of physical activity, reducing weight or body mass index (BMI), improving diet, improving blood pressure, blood glucose or blood lipid levels. Studies aimed at increasing physical health monitoring or access to physical healthcare which may improve cardiovascular health would also be included.

\section{Outcomes}

Primary outcome

Cardiovascular health, measured by 10-year CVD risk, cardiovascular mortality, number of cardiovascular events such as myocardial infarction or stroke, or any relevant measure of cardiovascular health quoted by the study.

Secondary outcomes

1. Measures of cardiovascular risk factors such as change in smoking status, change in levels of physical activity, change in weight or BMI, change in diet, blood pressure, blood glucose or blood lipid levels.

2. Leaving the study early for any reason including inefficacy of treatment, adverse events and death.

\section{Databases}

The following electronic databases were searched using NICE HDAS (Healthcare Databases Advanced Search): PsycINFO, MEDLINE and EMBASE. The search terms used for PsycINFO are provided in "Appendix". ${ }^{2}$ To limit the results to RCTs, the Cochrane Highly Sensitive Search Strategies for identifying randomised trials were employed for each of PsycINFO, MEDLINE and EMBASE databases [32]. No date, language, document type or publication status limitations were applied to the search.

In addition, the Cochrane Central Register of Controlled Trials (CENTRAL) was searched via the Cochrane Library. The following clinical trials registries were also searched to identify any unpublished or ongoing trials: World Health Organisation International Clinical Trials Registry Platform (ICTRP), ISRCTN Registry, EU Clinical Trials Register (EudraCT) and ClinicalTrials.gov. The reference lists of any included papers would also have been examined.

\footnotetext{
$\overline{2}$ Full search strategies for each database are available from the authors upon request.
}

Table 1 Results of database and trial registry searches

\begin{tabular}{lc}
\hline Database & Results \\
\hline PsycINFO & 379 \\
MEDLINE & 618 \\
EMBASE & 538 \\
CENTRAL & 479 \\
Trial registries & 31 \\
Total & 2045 \\
\hline
\end{tabular}

\section{Data collection and analysis}

\section{Selection of studies}

Covidence, a web-based software platform designed to streamline the production of systematic reviews, was used to facilitate the selection of studies [33]. Electronic records identified from our search were imported to Covidence via Endnote X8 [34]. Two review authors (KH and $\mathrm{KB}$ ) independently screened all records identified from the search to identify potentially relevant papers for full-text review. Any disagreements were resolved by discussion among the two review authors; had this not been possible, it would have been further discussed with the senior author (PM).

\section{Data extraction and management}

In the event of relevant studies being identified, the two review authors planned to extract data from the included studies onto standardised forms.

\section{Assessment of risk of bias in included studies}

The two review authors planned to assess the risk of bias independently, using the Cochrane Risk of Bias tool [35]. Risk of bias level and trial quality would then have been noted in a 'Summary of findings' table.

\section{Results}

\section{Description of studies}

From our searches, 2045 electronic records were identified (Table 1).

Three hundred and five duplicates were removed by Covidence, leaving 1740 records for screening. One paper was obtained for full-text screening but was excluded after discussion between the three authors (KH, KB and PM) as, although a proportion of the study population had a diagnosis of PD, results for this group were not differentiated from the included individuals with other diagnoses. This published RCT, conducted by Knapen et al., described how 
Fig. 1 PRISMA flow diagram

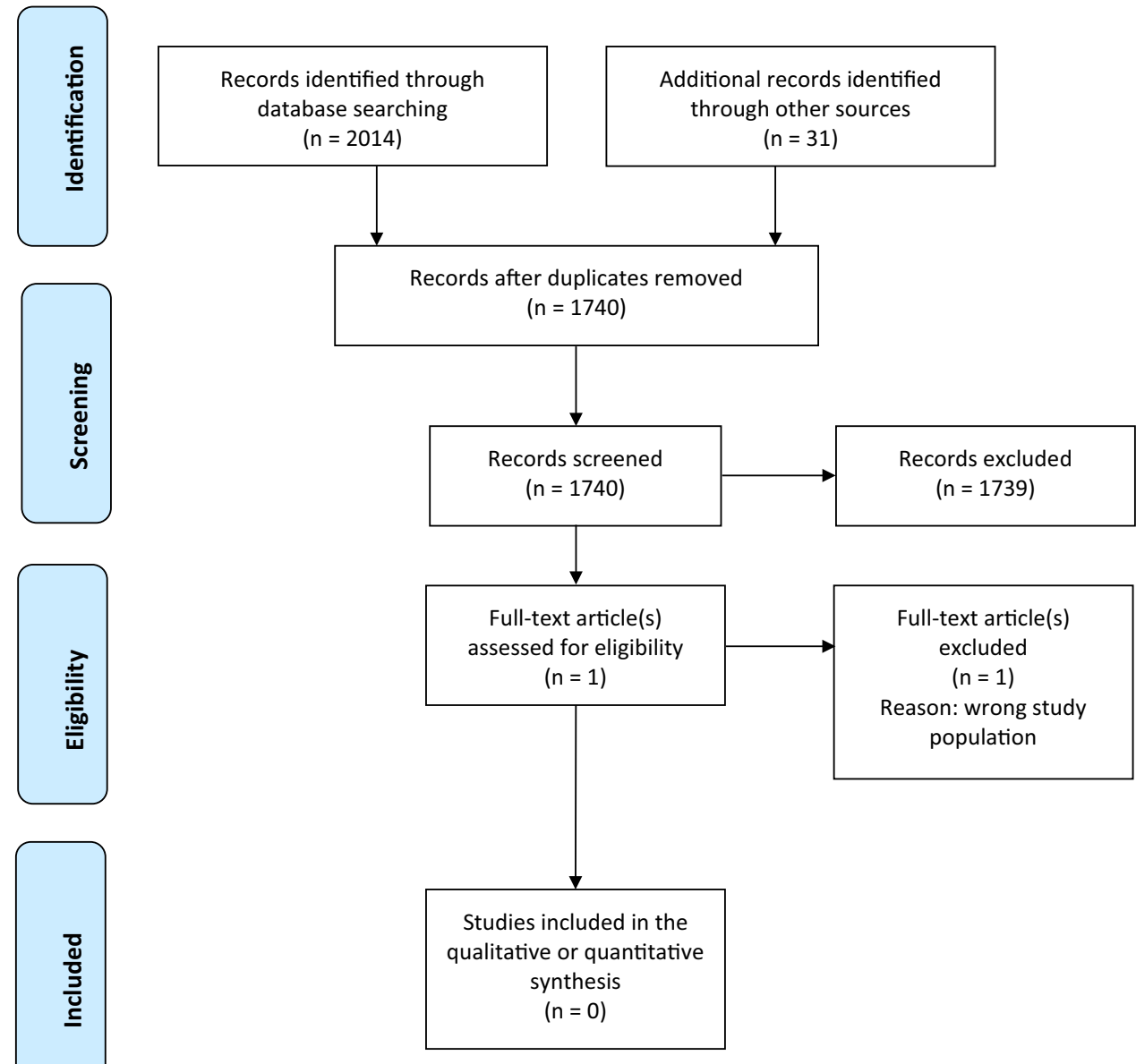

199 patients (70 of whom had a diagnosis of PD) were randomly allocated to either a general programme of psychomotor therapy (control) or a personalised psychomotor fitness programme (intervention), each lasting for 16 weeks. The intervention group showed an increase in cardiorespiratory fitness, but no subgroup analysis for the patients with PD was reported, or made available on request from the authors [36].

With regards to the trial registries search, no ongoing trials were identified which focused on people with PDs. As a result, no RCTs were eligible for inclusion in this review. This process has been summarised in a PRISMA flow diagram (Fig. 1).

\section{Discussion}

We have argued that the modifiable risk factors associated with the development of CVD constitute an important target for narrowing the life expectancy gap between people with PD and the general population. The lack of identified trials in this area may partly be explained by the fact that the association between PD and CVD is less widely known compared to the association between CVD and other serious mental illnesses (SMI) [37].

In contrast to the paucity of trials in patients with PD, we have identified 10 systematic reviews investigating interventions to improve the cardiovascular health or cardiovascular risk profile of people with SMI [38-47]. Moreover, most of these reviews did not include people with PD. We believe that excluding these people, who are at very high risk of CVD, is not only scientifically unsound but also represents a lost opportunity for developing an evidence base in a neglected field of health research.

Physical health appears to be under-assessed and undertreated in people with PD [48]. Sanatinia et al. (2015) found that a lower proportion of those in a random sample of people with PDs had documented evidence of smoking status, blood glucose and blood lipid levels compared to people with schizophrenia. Moreover, people with PD were less likely to be offered smoking cessation advice than those with schizophrenia [48].

Research in people with SMI may offer guidance on future productive trials for people with PD. If we take smoking as an example, several trials $(n=26)$ relate to smoking cessation in people with SMI, as shown by the recent 
review by Peckham et al. [39]. It is noteworthy that people with PD were excluded from this review. These trials often test relatively brief interventions, such as motivational interviewing. For example, Metse et al. conducted a cluster RCT of 754 adult psychiatric inpatients, $17.8 \%$ of whom had 'personality and other disorders' but with no further differentiation than this. They investigated the effect of a brief motivational interview and self-help material whilst in hospital, followed by a 4-month pharmacological and psychosocial intervention upon discharge. No significant effect was found on measures of prolonged abstinence at either 6 or 12 months, but intervention group participants were more likely than controls to have reduced cigarette consumption by $50 \%$ [49]. This suggests that there could be grounds for testing the effectiveness of a brief motivational intervention for smoking cessation among people with PD specifically.

The mode of treatment delivery is also important to consider for people with PD, many of whom may lead turbulent and chaotic lives. It has already been established that the telephone is a feasible and acceptable mode of delivering interventions for people with PD, such as the use of telephone coaching in the context of dialectical behavioural therapy (DBT) [50]. Telephone-delivered smoking cessation has been trialled in people with psychosis; for example, in a RCT involving 235 smokers with psychotic disorders, Baker et al. (2018) found that both an individual 'healthy lifestyle intervention' (consisting of motivational interviewing and cognitive behavioural therapy over 6 months) and telephonedelivered smoking cessation support achieved significant reductions in cardiovascular disease risk and smoking rates across a 36-month follow-up period [51]. Such telephonebased interventions do not yet appear to have been trialled to improve cardiovascular risk factors in people with PD. However, the telephone could be a potentially promising delivery platform for a brief intervention designed to promote smoking cessation among people with PD. Ben-Porath (2004) hypothesises that telephone coaching in the context of DBT and self-harm provides a framework for encouraging patients to seek help in 'more adaptive ways' than their usual coping mechanisms [50]; this may well be applicable in the context of smoking in people with PD.

Smoking cessation is one example, but inspiration may be derived from a variety of approaches. Other trials in people with mental illness have aimed to reduce weight or waist circumference, for example by attempting to change the 'obesogenic environment' of inpatient facilities, with some positive results in the short term though not the longer term [52]. Another example is the WebMOVE initiative in those with SMI, which consists of computerised weight-management with peer coaching, which contributed to a reduction in BMI in the intervention group [53]. Further studies aimed to improve CVD risk more generally through lifestyle coaching $[54,55]$, or to improve access to physical healthcare via a peer health navigator scheme [56]. These interventions may be of interest when planning future work in those with PD.

\section{Conclusion}

There is a conspicuous gap in the literature on interventions designed to improve the cardiovascular health of people with $\mathrm{PD}$, despite the significantly increased prevalence of CVDs and mortality in this population. The lack of studies in this field is particularly accentuated when compared to the extent of recent and ongoing research into physical health interventions for people with other types of psychiatric disorders, such as psychotic or affective illnesses.

It is important to strive for parity of healthcare within psychiatric practice and this is particularly the case for people with PD, who are so often still excluded from mainstream healthcare. Our review shows that they are also excluded from trials evaluating the effectiveness of cardiovascular interventions. We believe that this is magnifying a serious public health problem, impacting both the quality of life of individuals with PDs and adversely affecting productivity in the context of wider health economics. We would argue that people with PD should be included in future trials of cardiovascular interventions. Such research could help to inform improvements in general health and mortality rates for people with this common and complex mental health disorder.

Acknowledgements Thanks to Stephen Walker, Avon and Wiltshire Partnership Trust Librarian, and Sarah Herring, University of Bristol Medical Subject Librarian, for their help in developing the search strategies for this systematic review.

\section{Compliance with ethical standards}

This study was supported by the NIHR Biomedical Research Centre at University Hospitals Bristol NHS Foundation Trust and the University of Bristol. The views expressed in this publication are those of the author(s) and not necessarily those of the NHS, the National Institute for Health Research or the Department of Health and Social Care.

Conflict of interest The authors declare that they have no conflict of interest.

Open Access This article is distributed under the terms of the Creative Commons Attribution 4.0 International License (http://creativeco mmons.org/licenses/by/4.0/), which permits unrestricted use, distribution, and reproduction in any medium, provided you give appropriate credit to the original author(s) and the source, provide a link to the Creative Commons license, and indicate if changes were made. 


\section{Appendix: Example search strategy (PsycINFO)}

((exp "PERSONALITY DISORDERS"/OR ("personality disorder*").ti,ab OR ("personality patholog*").ti,ab OR ("personality difficult*").ti,ab OR (“disordered personalit*").ti,ab OR ("borderline personalit*" OR "antisocial personalit*" OR "avoidant personalit*" OR "dependent personalit*" OR "histrionic personalit*" OR "narcissistic personalit*" OR "obsessive compulsive personalit*" OR "paranoid personalit*" OR "passive aggressive personalit*" OR "schizoid personalit*" OR "schizotypal personalit*"). ti,ab OR (ICD AND (F60 OR F61 OR F62)).ti,ab OR (DSM AND (axis AND II)).ti,ab) AND (exp "CARDIOVASCULAR DISORDERS"/OR exp "CARDIOVASCULAR SYSTEM"/OR exp "CARDIOLOGY"/OR exp "METABOLIC SYNDROME"/OR ("cardiovascular" OR "cardiometabolic" OR "metaboli*" OR "myocardial" OR "heart disease" OR "stroke" OR "cerebrovascular" OR "ischaemi*" OR "ischemi*" OR "infarct*").ti,ab OR exp "TOBACCO SMOKING"/OR exp "SMOKING CESSATION"/OR ("smok*" OR "cigarette*").ti,ab OR exp DIETS/OR exp "DIETARY RESTRAINT"/OR exp "EATING BEHAVIOR"/OR exp "HEALTH BEHAVIOR"/OR exp NUTRITION/OR ("diet*” OR "healthy eating").ti,ab OR exp OBESITY/OR exp OVERWEIGHT/OR exp "WEIGHT LOSS"/ OR exp "WEIGHT CONTROL"/OR ("obes*” OR “overweight" OR "waist circumference").ti,ab OR exp "PHYSICAL ACTIVITY"/OR exp "PHYSICAL FITNESS"/OR ("exercis*" OR "fitness" OR "physical activit*").ti,ab OR exp "DIABETES MELLITUS"/OR exp "HYPERGLYCEMIA"/OR ("diabet*" OR "glucose" OR "hyperglycaemi*" OR "hyperglycemi*”).ti,ab OR exp HYPERTENSION/OR ("hypertens*" OR "blood pressure").ti,ab OR ("cholesterol" OR "hypercholesterolaemi*" OR "hypercholesterolaemi*" OR "lipid*" OR "hyperlipidaemi*" OR "hyperlipidemi*”). ti,ab)) AND (exp "TREATMENT EFFECTIVENESS EVALUATION"/OR ("random*” OR “control*”).ti,ab)

\section{References}

1. Huang Y, Kotov R, de Girolamo G, Preti A, Angermeyer M, Benjet $C$ et al (2009) DSM-IV personality disorders in the WHO World Mental Health Surveys. Br J Psychiatry 195(1):46-53

2. Newton-Howes G, Tyrer P, Anagnostakis K, Cooper S, BowdenJones O, Weaver T et al (2010) The prevalence of personality disorder, its comorbidity with mental state disorders, and its clinical significance in community mental health teams. Soc Psychiatry Psychiatr Epidemiol 45(4):453-460

3. Tyrer P, Reed GM, Crawford MJ (2015) Classification, assessment, prevalence, and effect of personality disorder. Lancet 385(9969):717-726
4. Fok ML, Hayes RD, Chang CK, Stewart R, Callard FJ, Moran P (2012) Life expectancy at birth and all-cause mortality among people with personality disorder. J Psychosom Res 73(2):104-107

5. Ajetunmobi O, Taylor M, Stockton D, Wood R (2013) Early death in those previously hospitalised for mental healthcare in Scotland: a nationwide cohort study, 1986-2010. BMJ Open 3(7)

6. Cailhol L, Francois M, Thalamas C, Garrido C, Birmes P, Pourcel L et al (2016) Is borderline personality disorder only a mental health problem? Personal Ment Health 10(4):328-336

7. Henderson M, Hotopf M, Shah I, Hayes RD, Kuh D (2011) Psychiatric disorder in early adulthood and risk of premature mortality in the 1946 British Birth Cohort. BMC Psychiatry 11:37

8. Dixon-Gordon KL, Conkey LC, Whalen DJ (2017) Recent advances in understanding physical health problems in personality disorders. Curr Opin Psychol 21:1-5

9. Frankenburg FR, Zanarini MC (2004) The association between borderline personality disorder and chronic medical illnesses, poor health-related lifestyle choices, and costly forms of health care utilization. J Clin Psychiatry 65(12):1660-1665

10. Keuroghlian AS, Frankenburg FR, Zanarini MC (2013) The relationship of chronic medical illnesses, poor health-related lifestyle choices, and health care utilization to recovery status in borderline patients over a decade of prospective follow-up. J Psychiatr Res 47(10):1499-1506

11. Cailhol L, Francois M, Thalamas C, Garrido C, Birmes P, Pourcel $\mathrm{L}$ et al (2016) Is borderline personality disorder only a mental health problem? Personality Mental Health 10(4):328-336

12. Organization WH (2015) Cardiovascular diseases (CVDs). http://www.who.int/en/news-room/fact-sheets/detail/cardiovasc ular-diseases-(cvds)

13. Samuels J (2011) Personality disorders: epidemiology and public health issues. Int Rev Psychiatry 23(3):223-233

14. Chang CK, Hayes RD, Broadbent M, Fernandes AC, Lee W, Hotopf $\mathrm{M}$ et al (2010) All-cause mortality among people with serious mental illness (SMI), substance use disorders, and depressive disorders in southeast London: a cohort study. BMC Psychiatry 10:77

15. Fok M, Hotopf M, Stewart R, Hatch S, Hayes R, Moran P (2014) Personality disorder and self-rated health: a population-based cross-sectional survey. J Pers Disord 28(3):319-333

16. Grigoletti L, Perini G, Rossi A, Biggeri A, Barbui C, Tansella $\mathrm{M}$ et al (2009) Mortality and cause of death among psychiatric patients: a 20-year case-register study in an area with a community-based system of care. Psychol Med 39(11):1875-1884

17. Qin P, Hawton K, Mortensen PB, Webb R (2014) Combined effects of physical illness and comorbid psychiatric disorder on risk of suicide in a national population study. Br J Psychiatry 204(6):430-435

18. El-Gabalawy R, Katz LY, Sareen J (2010) Comorbidity and associated severity of borderline personality disorder and physical health conditions in a nationally representative sample. Psychosom Med 72(7):641-647

19. Quirk SE, El-Gabalawy R, Brennan SL, Bolton JM, Sareen J, Berk M et al (2015) Personality disorders and physical comorbidities in adults from the United States: data from the National Epidemiologic Survey on Alcohol and Related Conditions. Soc Psychiatry Psychiatr Epidemiol 50(5):807-820

20. Lee HB, Bienvenu OJ, Cho SJ, Ramsey CM, Bandeen-Roche K, Eaton WW et al (2010) Personality disorders and traits as predictors of incident cardiovascular disease: findings from the 23-year follow-up of the Baltimore ECA study. Psychosomatics 51(4):289-296

21. Moran P, Stewart R, Brugha T, Jenkins R, Bhugra D, Bebbington $P$ et al (2007) Personality disorder and cardiovascular disease: results from a National household survey. J Clin Psychiatry 68(1):69-74 
22. Pietrzak RH, Wagner JA, Petry NM (2007) DSM-IV personality disorders and coronary heart disease in older adults: results from the National Epidemiologic Survey on alcohol and related conditions. J Gerontol Ser B Psychol Sci Soc Sci 62(5):P295

23. Greggersen W, Rudolf S, Brandt PW, Schulz E, Fassbinder E, Willenborg B et al (2011) Intima-media thickness in women with borderline personality disorder. Psychosom Med 73(7):627-632

24. Kahl KG, Greggersen W, Schweiger U, Cordes J, Correll CU, Frieling H et al (2013) Prevalence of the metabolic syndrome in patients with borderline personality disorder: results from a cross-sectional study. Eur Arch Psychiatry Clin Neurosci 263(3):205-213

25. Powers AD, Oltmanns TF (2013) Borderline personality pathology and chronic health problems in later adulthood: the mediating role of obesity. Personal Disord 4(2):152-159

26. Moran P, Romaniuk H, Coffey C, Chanen A, Degenhardt L, Borschmann $\mathrm{R}$ et al (2016) The influence of personality disorder on the future mental health and social adjustment of young adults: a population-based, longitudinal cohort study. Lancet Psychiatry 3(7):636-645

27. Yusuf S, Reddy S, Ounpuu S, Anand S (2001) Global burden of cardiovascular diseases: Part II: variations in cardiovascular disease by specific ethnic groups and geographic regions and prevention strategies. Circulation 104(23):2855-2864

28. Soeteman DI, Hakkaart-van Roijen L, Verheul R, Busschbach JJ (2008) The economic burden of personality disorders in mental health care. J Clin Psychiatry 69(2):259-265

29. Barnicot K, Katsakou C, Marougka S, Priebe S (2011) Treatment completion in psychotherapy for borderline personality disorder: a systematic review and meta-analysis. Acta Psychiatr Scand 123(5):327-338

30. Quirk SE, Berk M, Chanen AM, Koivumaa-Honkanen H, Brennan-Olsen SL, Pasco JA et al (2016) Population prevalence of personality disorder and associations with physical health comorbidities and health care service utilization: a review. Personal Disord 7(2):136-146

31. Moher D, Liberati A, Tetzlaff J, Altman DG, Group P (2009) Preferred reporting items for systematic reviews and meta-analyses: the PRISMA statement. J Clin Epidemiol 62(10):1006-1012

32. Higgins J, Green S (eds) Cochrane handbook for systematic reviews of interventions version 5.1.0 [updated March 2011], Chapter 6.4.11.1: The Cochrane Collaboration; 2011. http://www. handbook.cochrane.org

33. Covidence systematic review software, Veritas Health Innovation, Melbourne, Australia. http://www.covidence.org

34. Clarivate Analytics (previously Thomson Reuters) New York, NY. 2017. Endnote X8 Referencing Software. http://endnote.com/

35. Higgins J, Green S (eds) (2011) Cochrane handbook for systematic reviews of interventions version 5.1.0 [updated March 2011], Chapter 8.5: The Cochrane Collaboration. http://www.handbook. cochrane.org

36. Knapen J, Van de Vliet P, Van Coppenolle H, David A, Peuskens J, Knapen K et al (2003) The effectiveness of two psychomotor therapy programmes on physical fitness and physical self-concept in nonpsychotic psychiatric patients: a randomized controlled trial. Clin Rehabil 17(6):637-647

37. De Hert M, Dekker JM, Wood D, Kahl KG, Holt RI, Möller HJ (2009) Cardiovascular disease and diabetes in people with severe mental illness position statement from the European Psychiatric Association (EPA), supported by the European Association for the Study of Diabetes (EASD) and the European Society of Cardiology (ESC). Eur Psychiatry 24(6):412-424

38. Naslund JA, Whiteman KL, McHugo GJ, Aschbrenner KA, Marsch LA, Bartels SJ (2017) Lifestyle interventions for weight loss among overweight and obese adults with serious mental illness: a systematic review and meta-analysis. Gen Hosp Psychiatry 47:83-102

39. Peckham E, Brabyn S, Cook L, Tew G, Gilbody S (2017) Smoking cessation in severe mental ill health: what works? an updated systematic review and meta-analysis. BMC Psychiatry. 17(1):252

40. Martin H, Beard S, Clissold N, Andraos K, Currey L (2017) Combined aerobic and resistance exercise interventions for individuals with schizophrenia: a systematic review. Mental Health Phys Act. $12: 147-155$

41. Teasdale SB, Ward PB, Rosenbaum S, Samaras K, Stubbs B (2017) Solving a weighty problem: systematic review and metaanalysis of nutrition interventions in severe mental illness. Br J Psychiatry 210(2):110-118

42. Vancampfort D, Rosenbaum S, Schuch F, Ward PB, Richards J, Mugisha J et al (2017) Cardiorespiratory fitness in severe mental illness: a systematic review and meta-analysis. Sports Med 47(2):343-352

43. Stubbs B, Williams J, Shannon J, Gaughran F, Craig T (2016) Peer support interventions seeking to improve physical health and lifestyle behaviours among people with serious mental illness: a systematic review. Int J Ment Health Nurs. 25(6):484-495

44. Olker SJ, Parrott JS, Swarbrick MA, Spagnol AB (2016) Weight management interventions in adults with a serious mental illness: a meta-analytic review. Am J Psychiatr Rehabil 19(4):370-393

45. Wu Q, Gilbody S, Peckham E, Brabyn S, Parrott S (2016) Varenicline for smoking cessation and reduction in people with severe mental illnesses: systematic review and meta-analysis. Addiction 111(9):1554-1567

46. Pearsall R, Thyarappa Praveen K, Pelosi A, Geddes J (2016) Dietary advice for people with schizophrenia. Cochrane Database Syst Rev 3:CD009547

47. Stubbs B, Rosenbaum S, Vancampfort D, Ward PB, Schuch FB (2016) Exercise improves cardiorespiratory fitness in people with depression: a meta-analysis of randomized control trials. J Affect Disord 190:249-253

48. Sanatinia R, Middleton SM, Lin T, Dale O, Crawford MJ (2015) Quality of physical health care among patients with personality disorder. Personal Mental Health. 9(4):319-329

49. Metse AP, Wiggers J, Wye P, Wolfenden L, Freund M, Clancy R et al (2017) Efficacy of a universal smoking cessation intervention initiated in inpatient psychiatry and continued post-discharge: a randomised controlled trial. Aust N Z J Psychiatry 51(4):366-381

50. Ben-Porath D (2004) Intersession telephone contact with individuals diagnosed with borderline personality disorder: lessons from dialectical behavior therapy. Cognit Behav Pract. 11(2):222-230

51. Baker AL, Richmond R, Kay-Lambkin FJ, Filia SL, Castle D, Williams JM et al (2018) Randomised controlled trial of a healthy lifestyle intervention among smokers with psychotic disorders: outcomes to 36 months. Aust N Z J Psychiatry 52(3):239-252

52. Looijmans A, Stiekema APM, Bruggeman R, van der Meer L, Stolk RP, Schoevers RA et al (2017) Changing the obesogenic environment to improve cardiometabolic health in residential patients with a severe mental illness: cluster randomised controlled trial. Br J Psychiatry 211(5):296-303

53. Young AS, Cohen AN, Goldberg R, Hellemann G, Kreyenbuhl J, Niv N et al (2017) Improving weight in people with serious mental illness: the effectiveness of computerized services with peer coaches. J Gen Intern Med 32(Suppl 1):48-55

54. Jakobsen AS, Speyer H, Nørgaard HCB, Karlsen M, Birk M, Hjorthøj C et al (2017) Effect of lifestyle coaching versus care coordination versus treatment as usual in people with severe mental illness and overweight: two-years follow-up of the randomized CHANGE trial. PLoS One 12(10):e0185881

55. Speyer H, Christian Brix Nørgaard H, Birk M, Karlsen M, Storch Jakobsen A, Pedersen K et al (2016) The CHANGE trial: no superiority of lifestyle coaching plus care coordination plus treatment 
as usual compared to treatment as usual alone in reducing risk of cardiovascular disease in adults with schizophrenia spectrum disorders and abdominal obesity. World Psychiatry. 15(2):155-165

56. Kelly E, Duan L, Cohen H, Kiger H, Pancake L, Brekke J (2017) Integrating behavioral healthcare for individuals with serious mental illness: a randomized controlled trial of a peer health navigator intervention. Schizophr Res 182:135-141 